\title{
Systematic Review \\ Additional Value of Patient-Reported Symptom Monitoring in Cancer Care: A Systematic Review of the Literature
}

\author{
Luís Lizán 1,2,* , Lucía Pérez-Carbonell ${ }^{2}$ and Marta Comellas ${ }^{2}$ \\ 1 Department of Medicine, Jaume I University, 12071 Castellón de la Plana, Spain \\ 2 Outcomes'10, Jaume I University, 12071 Castellón de la Plana, Spain; lperez@outcomes10.com (L.P.-C.); \\ mcomellas@outcomes10.com (M.C.) \\ * Correspondence: lizan@outcomes10.com; Tel.: +34-608-262-673
}

Citation: Lizán, L.; Pérez-Carbonell, L.; Comellas, M. Additional Value of Patient-Reported Symptom Monitoring in Cancer Care: A Systematic Review of the Literature. Cancers 2021, 13, 4615. https:// doi.org/10.3390/cancers13184615

Academic Editors: Massimo Di Maio and Marianne Jensen Hjermstad

Received: 31 July 2021

Accepted: 10 September 2021

Published: 15 September 2021

Publisher's Note: MDPI stays neutral with regard to jurisdictional claims in published maps and institutional affiliations.

Copyright: (c) 2021 by the authors. Licensee MDPI, Basel, Switzerland. This article is an open access article distributed under the terms and conditions of the Creative Commons Attribution (CC BY) license (https:/ / creativecommons.org/licenses/by/ $4.0 /)$.
Simple Summary: The additional value of patient-reported symptom monitoring in routine cancer is still under discussion. With this in mind, we have reviewed recent evidence on the benefits of this strategy. The evidence examined illustrates that bringing systematic patient feedback into the oncology consultation provides objective advantages over usual care, such as better symptom control, early detection of tumor recurrence, and extended chemotherapy use. Such care improvements ultimately entail an outstanding survival benefit for advanced cancer patients, an increase in their global quality of life, and eventually, medical cost savings. Monitoring patient-reported symptoms might also have other implications in clinical practice, such as promoting patient disease awareness or enhancing patient-physician communication and relationships. Notwithstanding these advantages, there are still logistical barriers that prevent its widespread implementation-especially in the electronic modality. In addition, the real-world effectiveness and the cost-effectiveness of this strategy are yet to be proven in different settings.

Abstract: Background: To describe the benefit of patient-reported symptom monitoring on clinical, other patient-reported, and economic outcomes. Methods: We conducted a systematic literature review using Medline/PubMed, limited to original articles published between 2011 and 2021 in English and Spanish, and focused on the benefit of patient-reported symptom monitoring on cancer patients. Results: We identified 16 reports that deal with the benefit of patient-reported symptom monitoring (collected mostly electronically) on different outcomes. Five studies showed that patient-reported symptom surveillance led to significantly improved survival compared with usual care-mainly through better symptom control, early detection of tumor recurrence, and extended chemotherapy use. Additionally, three evaluations demonstrated an improvement in Health-Related Quality of Life (HRQoL) associated with this monitoring strategy, specifically by reducing symptom severity. Additionally, six studies observed that this monitoring approach prevented unplanned emergency room visits and hospital readmissions, leading to a substantial decrease in healthcare usage. Conclusions: There is consistent evidence across the studies that patient-reported symptom monitoring might entail a substantial survival benefit for cancer patients, better HRQoL, and a considerable decrease in healthcare usage. Nonetheless, more studies should be conducted to demonstrate their effectiveness in addition to their cost-effectiveness in clinical practice.

Keywords: patient-reported symptoms; survival; Health-Related Quality of Life; satisfaction; use of resources

\section{Introduction}

Cancer patients suffer from significant physical and psychosocial consequences derived from either the disease itself or cancer treatment toxicities [1]. For years, physicians have mainly focused on traditional oncological outcomes such as overall or disease-free survival, and tumor response, to evaluate and monitor cancer patients. In contrast, they 
might have overlooked cancer-related symptoms such as pain, nausea, or headaches [2-4], or other consequences of cancer such as distress and fatigue [5]. Underrecognizing and undertreating cancer-related symptoms might directly affect the continuity of cancer treatment. In addition, it might increase cancer morbidity or the need for healthcare resources, thus adding substantial medical costs [6].

Information about physical symptoms such as pain, nausea, and psychological consequences (anxiety, depression, or sleep disturbances) can only be obtained directly from patients. Thus, the use of the Patient-Reported Outcomes (PROs) assessment during cancer patients' follow-up may provide a more complete picture of their general health. In addition, the development of technology might facilitate the collection of these PROs through computers, tablets, or smartphones so that patients can report their symptoms during and between visits. Moreover, at the same time, healthcare providers can receive real-time alerts in case of clinical deterioration, whereby they can respond rapidly [7]. According to different reviews, gathering patient-reported symptoms (including physical symptoms and psychological disturbances) during cancer patients' follow-up may lead to an improvement in patient management and symptom control [8,9], may improve patient satisfaction with treatment $[9,10]$, and may improve the communication between clinicians and their patients $[10,11]$.

Despite the growing interest in patient-reported symptom monitoring, especially in the electronic modality, there is still much debate as to its additional value in routine cancer follow-up. Indeed, there is insufficient understanding of the impact of patientreported symptom monitoring on health outcomes [10-12]. Therefore, this systematic review aimed to describe the benefits of patient-reported symptom monitoring-regardless of the modality (paper-based or electronic)—on health outcomes such as clinical (e.g., survival), patient-reported (e.g., Health-Related Quality of Life [HRQoL], general perception or feelings of well-being, satisfaction, etc.) and economic outcomes (use of healthcare resources, costs, cost-effectiveness, etc.).

\section{Materials and Methods}

\subsection{Search Design}

We conducted a systematic review of the literature according to the Cochrane methodology [13] and the Preferred Reporting Items for Systematic Reviews and Meta-Analyses (PRISMA) checklist for reporting [14] (see Tables S1 and S2). The protocol for our systematic review was registered in the search registry database (reviewregistry1221). We acknowledge that a large amount of evidence regarding PROs and cancer has emerged in recent years; thus, we developed a search strategy to maximize the identification of recent literature about the benefits of patient-reported symptom monitoring as an intervention, but we tried to find the best balance between sensitivity and specificity for retrieving publications. To do so, we searched the terms related to the population (cancer) and the intervention (PROs and patient-reported symptoms) as free-text keywords in the title field. Then, we narrowed the search adding terms related to the outcomes, using either free-text or $\mathrm{MeSH}$ terms when available. Additionally, we avoided overlapping terms, that is, those that do not add new results to the search. Terms were combined with Boolean operators (AND/OR), and the search was adapted to the Medline/PubMed international database. Tables S3 and S4 show the research strategy in more details. Additionally, we manually searched the reference lists of relevant original articles and reviews obtained in the search. We also carried out a search of the grey literature using the search engine Google Scholar and combined text terms such as patient reported-symptoms, survival, satisfaction, adherence, resources, and costs. Furthermore, we reviewed international congress pages related to outcome research and pharmacoeconomics, such as the International Society for Pharmacoeconomics and Outcomes Research (ISPOR).

The search was limited to original articles published in the last ten years (2011-2021) in English or Spanish, conducted in Europe, North America, and Australia, focusing on (1) the use of patient-reported symptom monitoring in the context of clinical trials or routine 
practice in cancer patients, and (2) the impact of patient-reported symptom monitoring on health outcomes, including clinical (e.g., survival), patient-reported (e.g., HRQoL, general perception or feelings of well-being, satisfaction, etc.) or economic outcomes (use of healthcare resources, costs, cost-effectiveness, etc.).

\subsection{Data Abstraction and Quality Assessment}

Two researchers independently screened each of the identified publications based on their titles, abstracts, and full texts for inclusion criteria. Any discrepancies between reviewers were resolved through consensus and, if necessary, by consulting a third reviewer. Table 1 shows the inclusion criteria following the PICOS (population, intervention, comparator, outcomes, and study design) definition.

Table 1. Eligibility criteria defined by PICOS.

\begin{tabular}{|c|c|}
\hline \multicolumn{2}{|r|}{ Inclusion Criteria } \\
\hline Population & Cancer patients (any type and stage) \\
\hline Intervention & $\begin{array}{l}\text { Patient-reported symptom monitoring, regardless of the } \\
\text { modality: paper-based or electronic }\end{array}$ \\
\hline Comparator & - \\
\hline Outcome & $\begin{array}{l}\text { Health results as clinical (e.g., survival), patient-reported (e.g., } \\
\text { HRQoL, general perception or feelings of well-being, } \\
\text { satisfaction), or economic (use of healthcare resources, costs, } \\
\text { cost-effectiveness) outcomes }\end{array}$ \\
\hline Study Design & Original articles \\
\hline
\end{tabular}

From the final selected articles, we extracted the following variables: first author; year of publication; region (country); study design (e.g., observational study or clinical trial); the existence of comparators (e.g., only one arm, or two arms); cancer type and sample size (e.g., metastatic cancer or advanced lung cancer); list of patient-reported outcomes assessed (e.g., patient-reported physical symptoms and/or psychological consequences); instruments used for patient-reported symptom monitoring (e.g., the Edmonton Symptom Assessment System [ESAS]); when (frequency) and how patient-reported symptoms were collected (electronically via a web platform, or using a mobile application [e.g., alert email to the treatment oncologist]); health outcomes measured to evaluate the impact of patientreported symptom collection; summary of results; main article conclusion; and quality of the study.

Two researchers independently assessed the quality of the studies reviewed against the 30 points of the CONSORT statement for clinical trials [15], the 22 essential points of the STROBE declaration for observational studies [16], and the 24 points of the Consolidated Health Economic Evaluation Reporting Standards (CHEERS) checklist for the reporting of economic evaluations [17]. To unify the costs reported from different studies, costs were converted into Spanish euros (2021) using the CCEMG-EPPI center Cost Converter tool [18].

\section{Results}

\subsection{General Results}

We first identified 1248 studies in MedLine/PubMed and two studies in sources identified via Google Scholar. Of these, 1190 were excluded as they did not provide relevant information for the purpose of the review. We assessed 60 full-text articles for eligibility (Figure 1) and identified 16 publications that discussed the impact of integrating patient-reported symptom monitoring into cancer care. Table 2 shows the summary of the studies included. Table S5 specifies the articles excluded and the reasons for their exclusion: the most common reasons for exclusion were that the publications did not assess the 
impact of patient-reported symptom monitoring on health outcomes $(n=21 / N=44)$ and they focused on the feasibility and utility of patient-reported outcome measures (PROMs) $(n=10 / N=44)$.

In short, we identified 16 publications from 13 different studies: three of them published results from the same clinical trial reported by Basch et al. [19-21], whereas one economic evaluation [22] was a pre-specified secondary outcome of the Denis et al. [23] trial. All the studies were implemented in Europe and North America (ten of them in the United States and Canada). Of the 16 studies identified, eight had a randomized clinical trial design [19-21,23-27], six were observational studies [28-33] and two were economic evaluations [22,34]. Of the clinical trials, five reports [20,24-27] were randomized controlled trials with a high-quality design, whereas two $[19,23]$ were research letters that communicated brief reports of data. Finally, Nipp et al. [21] reported a secondary analysis based on a randomized clinical trial [20]. Among the observational studies, one had a retrospective matched cohort design [28], whereas four used a prospective-cohort design to examine the consequences of implementing a patient-reported symptom strategy in clinical practice [30-33]. The remaining study compared a patient-reported symptom strategy prospectively with a historical cohort [29].

All the studies except for one [32] examined the impact of patient-reported symptom monitoring compared to usual care monitoring. In the studies reviewed, usual care typically involved patients and their oncologists discussing symptoms during scheduled visits, whereas patient-reported symptom monitoring involved patients regularly self-reporting cancer-related symptoms. In most cases $(n=13)$, patient-reported symptoms were collected electronically through websites, mobile applications, touch screen kiosks, or tablets during and between scheduled visits [19-23,25-28,30,32-34].

In six studies [19-23,34], the approach included email alerts sent to the treating physician when patient-reported symptoms matched predefined criteria for severity. These alerts enabled clinicians to take rapid clinical actions in response to these reports.

The most frequently monitored physical cancer-related symptoms were pain $(n=16)$, loss of appetite $(n=14)$, and nausea $(n=13)$, whereas only six out of the 16 studies assessed depression symptoms.

Twelve studies addressed patients with different types of cancers [19-21,24,25,27$31,33,34]$, and eight of them targeted advanced or metastatic cancer patients [19-21,24, $25,27,29,34]$. Four publications included specific types of cancers [22,23,26,32], most of which evaluated the impact of patient-reported symptom monitoring on lung cancer patients $[22,23,32]$. 


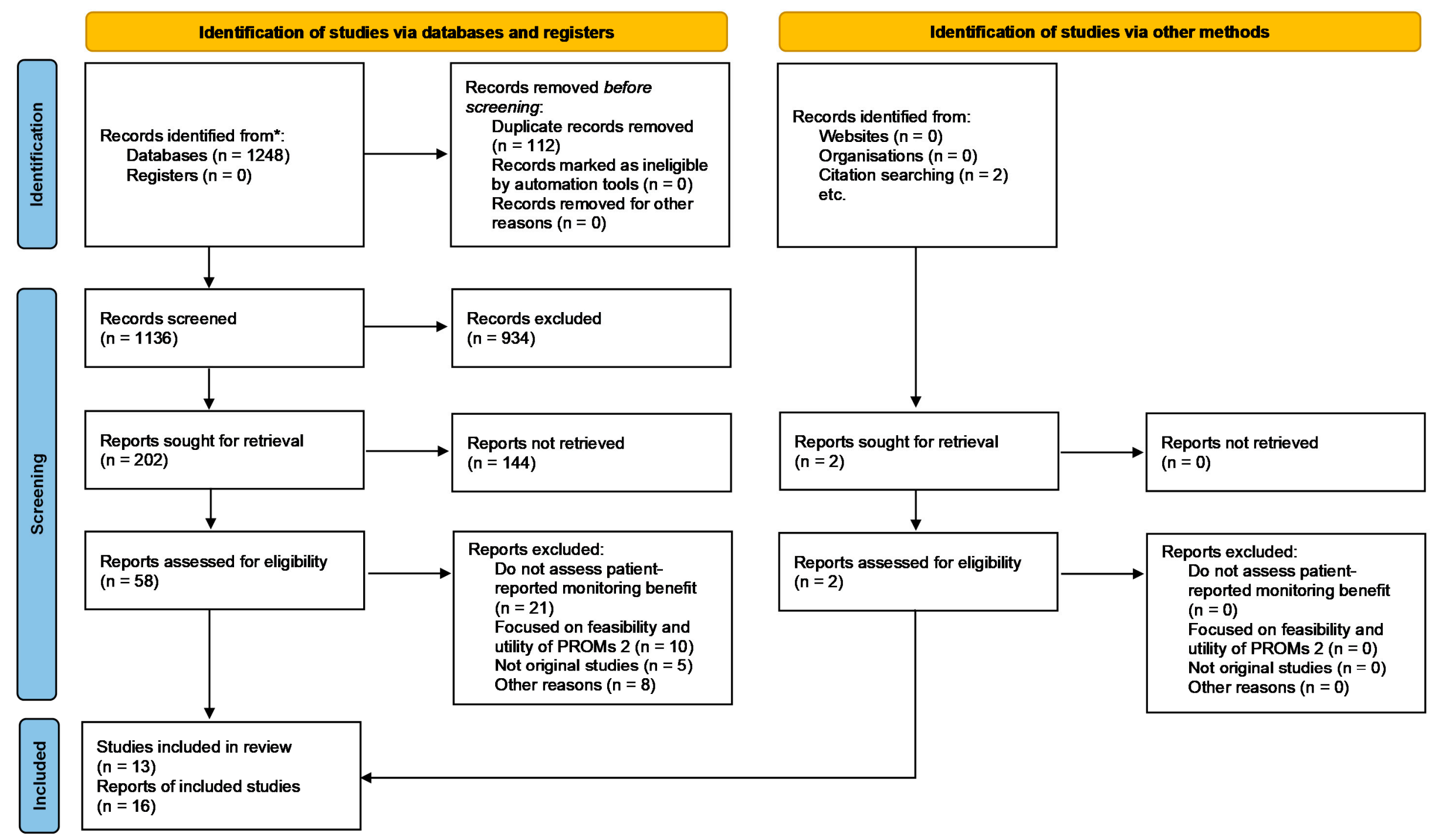

Figure 1. PRISMA flow diagram. * MedLine/PubMed database. 
Table 2. Summary of the identified studies.

\begin{tabular}{|c|c|c|c|c|c|c|c|c|}
\hline $\begin{array}{l}\text { Author and Year of } \\
\text { Publication, Country }\end{array}$ & $\begin{array}{l}\text { Type of Study (a), } \\
\text { Comparators (b) }\end{array}$ & Study Aim & $\begin{array}{l}\text { Cancer Type; } \\
\text { Sample Size }\end{array}$ & $\begin{array}{c}\text { Patient-Reported } \\
\text { Symptoms Assessed, } \\
\text { Instrument Used and } \\
\text { Frequency }\end{array}$ & $\begin{array}{c}\text { Type of Outcome } \\
\text { Evaluated }\end{array}$ & Summary of Results & Main Conclusion & Study Quality \\
\hline $\begin{array}{c}\text { Barbera et al. [28], 2020, } \\
\text { Canada }\end{array}$ & $\begin{array}{l}\text { (a) Observational } \\
\text { retrospective cohort. } \\
\text { (b) Cancer patients } \\
\text { exposed (patients must } \\
\text { have completed at least } \\
\text { one assessment during } \\
\text { the study) vs. not } \\
\text { exposed to ESAS }\end{array}$ & $\begin{array}{l}\text { To examine the effect of } \\
\text { ESAS exposure on } \\
\text { cancer patients' overall } \\
\text { survival }\end{array}$ & $\begin{array}{c}\text { Different types of } \\
\text { cancer (most prevalent: } \\
\text { prostate [17.4\%], breast } \\
\text { [14.5\%] and } \\
\text { hematology [13.4\%]). } \\
N=257,786 \text { ( } n=128,893 \\
\text { patients exposed to } \\
\text { ESAS matched to } 128 \\
893 \text { cancer patients not } \\
\text { exposed). }\end{array}$ & $\begin{array}{l}\text { ESAS symptoms (pain, } \\
\text { tiredness, nausea, } \\
\text { depression, anxiety, } \\
\text { drowsiness, appetite } \\
\text { loss, well-being, and } \\
\text { shortness of breath). } \\
\text { Collected before a } \\
\text { patient's visit via a } \\
\text { touch screen kiosk and } \\
\text { discussed during the } \\
\text { clinical encounter. }\end{array}$ & $\begin{array}{l}\text { Clinical outcome: } \\
\text { probability of survival } \\
\text { at } 1,3 \text {, and } 5 \text { years. }\end{array}$ & $\begin{array}{c}\text { Probability of survival } \\
\text { at } 1 \text { year: } 81.9 \% \text { vs. } \\
76.4 \% \\
\text { Probability of survival } \\
\text { at } 3 \text { years: } 68.3 \% \text { vs. } \\
66.1 \% \\
\text { Probability of survival } \\
\text { at } 5 \text { years: } 61.9 \% \text { vs. } \\
61.4 \% \\
\text { All comparisons } \\
p<0.0001\end{array}$ & $\begin{array}{l}\text { Patients' exposure to } \\
\text { ESAS collection is } \\
\text { associated with } \\
\text { improved survival in } \\
\text { cancer patients }\end{array}$ & 17/22 (STROBE) \\
\hline $\begin{array}{l}\text { Basch et al. [19], 2017, } \\
\text { USA (research letter) }\end{array}$ & $\begin{array}{l}\text { (a) Randomized clinical } \\
\text { trial. } \\
\text { (b) Patients at } \\
\text { patient-reported } \\
\text { symptom monitoring } \\
\text { vs. usual care }\end{array}$ & $\begin{array}{l}\text { To compare overall } \\
\text { survival associated } \\
\text { with electronic } \\
\text { patient-reported } \\
\text { symptom monitoring } \\
\text { vs. usual care in cancer } \\
\text { patients }\end{array}$ & $\begin{array}{c}\text { Cancer patients } \\
\text { initiating } \\
\text { chemotherapy for solid } \\
\text { metastatic tumors } \\
\text { (various types). } \\
N=766 \text { patients } \\
(n=441 \text { intervention } \\
\text { arm vs. } n=325 \text { control } \\
\text { arm). }\end{array}$ & $\begin{array}{l}\text { Twelve symptoms } \\
\text { (appetite loss, } \\
\text { constipation, cough, } \\
\text { diarrhea, dyspnea, } \\
\text { dysuria, fatigue, hot } \\
\text { flashes, nausea, pain, } \\
\text { neuropathy, and } \\
\text { vomiting) } \\
\text { Symptoms were } \\
\text { collected at or between } \\
\text { visits via a web-based } \\
\text { questionnaire platform } \\
\text { (computer-experienced } \\
\text { patients) or free } \\
\text { standing. } \\
\text { computer kiosks } \\
\text { (computer- } \\
\text { inexperienced patients). } \\
\text { The system included } \\
\text { email alerts to the } \\
\text { treating oncologist }\end{array}$ & $\begin{array}{c}\text { Clinical outcome: } \\
\text { median overall survival }\end{array}$ & $\begin{array}{c}\text { Median follow-up: } \\
7 \text { years (IQR, 6.5-7.8) } \\
\text { Median overall } \\
\text { Survival: } 31.2 \text { months } \\
\text { (95\% CI, } 24.5-39.6 \text { ) vs. } \\
26.0 \text { months (95\% CI, } \\
22.1-30.9 \text { ) (difference, } \\
5.2 \text { months; } p=0.03 \text { ) }\end{array}$ & $\begin{array}{c}\text { Integration of } \\
\text { patient-reported } \\
\text { symptoms into the } \\
\text { routine care of patients } \\
\text { with metastatic cancer } \\
\text { was associated with } \\
\text { increased survival } \\
\text { compared with usual } \\
\text { care. }\end{array}$ & - \\
\hline $\begin{array}{l}\text { Denis et al. [23], 2019, } \\
\text { France (research letter) }\end{array}$ & $\begin{array}{c}\text { (a) Randomized clinical } \\
\text { trial. } \\
\text { (b) Patient-reported } \\
\text { symptom monitoring } \\
\text { vs. usual care }\end{array}$ & $\begin{array}{l}\text { To compare overall } \\
\text { survival associated } \\
\text { with electronic } \\
\text { patient-reported } \\
\text { symptom monitoring } \\
\text { vs. usual care in cancer } \\
\text { patients }\end{array}$ & $\begin{array}{l}\text { Patients with advanced } \\
\text { nonprogressive stages } \\
\text { IIA to IV lung cancer } \\
N=121 \text { patients }(n=60 \\
\text { intervention arm vs. } \\
n=61 \text { control arm) }\end{array}$ & $\begin{array}{l}\text { Thirteen symptoms } \\
\text { (weight, weight } \\
\text { variation, appetite loss, } \\
\text { weakness, pain, cough, } \\
\text { breathlessness, } \\
\text { depression, fever, face } \\
\text { swelling, lump under } \\
\text { skin, voice changing, } \\
\text { blood in sputum). } \\
\text { Collected weekly in an } \\
\text { electronic form } \\
\text { between visits. The } \\
\text { approach included an } \\
\text { alert email to the } \\
\text { treating oncologist. }\end{array}$ & $\begin{array}{l}\text { Clinical outcome: } \\
\text { median overall survival }\end{array}$ & $\begin{array}{l}\text { Two years of follow-up } \\
\text { Median overall } \\
\text { survival: } 22.5 \text { months } \\
\text { vs. } 14.9 \text { months } \\
\text { (difference, } 7.6 \text { months; } \\
\text { HR: } 0.59 \text { ( } 95 \% \text { CI } \\
0.37-0.96) ; p=0.03 \text { ) }\end{array}$ & $\begin{array}{l}\text { Symptom monitoring } \\
\text { via weekly web-based } \\
\text { patient-reported } \\
\text { symptom monitoring } \\
\text { treatment for lung } \\
\text { cancer was associated } \\
\text { with increased survival } \\
\text { compared with } \\
\text { standard imaging } \\
\text { surveillance. }\end{array}$ & - \\
\hline
\end{tabular}


Table 2. Cont.

\begin{tabular}{|c|c|c|c|c|c|c|c|c|}
\hline $\begin{array}{l}\text { Author and Year of } \\
\text { Publication, Country }\end{array}$ & $\begin{array}{l}\text { Type of Study (a), } \\
\text { Comparators (b) }\end{array}$ & Study Aim & $\begin{array}{l}\text { Cancer Type; } \\
\text { Sample Size }\end{array}$ & $\begin{array}{l}\text { Patient-Reported } \\
\text { Symptoms Assessed, } \\
\text { Instrument Used and } \\
\text { Frequency }\end{array}$ & $\begin{array}{l}\text { Type of Outcome } \\
\text { Evaluated }\end{array}$ & Summary of Results & Main Conclusion & Study Quality \\
\hline $\begin{array}{l}\text { Basch et al. [20], 2016, } \\
\text { USA }\end{array}$ & $\begin{array}{l}\text { (a) Randomized } \\
\text { nonblinded, clinical } \\
\text { trial. } \\
\text { (b) Web-based } \\
\text { self-reporting of } \\
\text { symptoms vs. usual } \\
\text { care. }\end{array}$ & $\begin{array}{l}\text { To test whether } \\
\text { systematic web-based } \\
\text { collection of } \\
\text { patient-reported } \\
\text { symptoms during } \\
\text { chemotherapy } \\
\text { treatment improves } \\
\text { HRQoL and survival, } \\
\text { quality-adjusted } \\
\text { survival, emergency } \\
\text { room use, and } \\
\text { hospitalization. }\end{array}$ & $\begin{array}{l}\text { Patients with metastatic } \\
\text { breast, genitourinary, } \\
\text { gynecologic, or lung } \\
\text { cancer-initiating } \\
\text { chemotherapy. } \\
N=766 \text { patients } \\
(n=411 \text { intervention } \\
\text { arm vs. } n=325 \text { in } \\
\text { control arm) }\end{array}$ & $\begin{array}{l}\text { Twelve symptoms } \\
\text { (appetite loss, } \\
\text { constipation, cough, } \\
\text { diarrhea, dyspnea, } \\
\text { dysuria, fatigue, hot } \\
\text { flashes, nausea, pain, } \\
\text { neuropathy, and } \\
\text { vomiting) } \\
\text { Symptoms collected at } \\
\text { or between visits via a } \\
\text { web-based } \\
\text { questionnaire platform } \\
\text { (computer-experienced } \\
\text { patients) or } \\
\text { free-standing computer } \\
\text { kiosks (computer- } \\
\text { inexperienced patients). } \\
\text { The system included } \\
\text { email alerts to the } \\
\text { treating oncologist. }\end{array}$ & $\begin{array}{l}\text { Patient-reported } \\
\text { outcome: percentage of } \\
\text { patients with HRQoL } \\
\text { clinically meaningful } \\
\text { improvement } \\
\text { ( } \geq 6 \text { points) at six } \\
\text { months (EuroQol } \\
\text { EQ-5D Index) } \\
\text { Use of resources: } \\
\text { percentage of patients } \\
\text { who visit the } \\
\text { emergency room and } \\
\text { who were hospitalized. } \\
\text { Time receiving active } \\
\text { cancer } \\
\text { treatment. } \\
\text { Clinical outcome: } \\
\text { percentage of patients } \\
\text { alive at one year }\end{array}$ & $\begin{array}{c}\text { Patient-reported } \\
\text { outcome (HRQoL } \\
\text { improvement } \\
\text { [ } \geq 6 \text { points]): } 21 \% \text { vs. } \\
11 \%(p<0.001) \\
\text { Use of resources and: } \\
\text { visits to the emergency } \\
\text { room ( } 34 \% \text { vs. } 41 \% ; \\
p=0.02) \\
\text { hospitalizations }(45 \% \\
\text { vs. } 49 \% ; p=0.08) \text {, time } \\
\text { receiving treatment } \\
\text { (mean of } 8.2 \text { months } \\
\text { (range } 0-49) \text { vs. } \\
6.3 \text { months (range } \\
0-41), \\
\text { respectively }(p=0.002) \\
\text { Clinical outcome: } 75 \% \\
\text { vs. } 69 \% \text { patients alive } \\
\text { at one year (difference } \\
6 \% ; p=0.05 \text { ) }\end{array}$ & $\begin{array}{l}\text { Symptom } \\
\text { self-reporting engages } \\
\text { patients as active } \\
\text { participants and may } \\
\text { improve the experience, } \\
\text { efficiency, and } \\
\text { outcomes of care }\end{array}$ & 30/30 (CONSORT) \\
\hline
\end{tabular}


Table 2. Cont.

\begin{tabular}{|c|c|c|c|c|c|c|c|c|}
\hline $\begin{array}{l}\text { Author and Year of } \\
\text { Publication, Country }\end{array}$ & $\begin{array}{l}\text { Type of Study (a), } \\
\text { Comparators (b) }\end{array}$ & Study Aim & $\begin{array}{l}\text { Cancer Type; } \\
\text { Sample Size }\end{array}$ & $\begin{array}{c}\text { Patient-Reported } \\
\text { Symptoms Assessed, } \\
\text { Instrument Used and } \\
\text { Frequency }\end{array}$ & $\begin{array}{l}\text { Type of Outcome } \\
\text { Evaluated }\end{array}$ & Summary of Results & Main Conclusion & Study Quality \\
\hline $\begin{array}{c}\text { Patel et al. [29], 2019, } \\
\text { USA }\end{array}$ & $\begin{array}{l}\text { (a) Observational study. } \\
\text { Cancer registry } \\
\text { (prospective and } \\
\text { retrospective design) } \\
\text { (b) Usual care } \\
\text { (historical cohort) vs. } \\
\text { Lay } \\
\text { health worker } \\
\text { (LHW)-led symptom } \\
\text { screening intervention }\end{array}$ & $\begin{array}{l}\text { To evaluate the effect of } \\
\text { a } \\
\text { LHW-led symptom } \\
\text { screening intervention } \\
\text { on satisfaction, } \\
\text { self-reported overall } \\
\text { and mental } \\
\text { health, health care use, } \\
\text { total costs, and } \\
\text { survival. }\end{array}$ & $\begin{array}{c}\text { Patients with stage } 3 \text { or } \\
4 \text { solid tumors or } \\
\text { hematologic } \\
\text { malignancies who were } \\
\text { receiving } \\
\text { care in a community } \\
\text { oncology practice (most } \\
\text { prevalent: } \\
\text { gastrointestinal }[27.4 \%], \\
\text { breast [20.5\% } \% \text { and } \\
\text { genitourinary [16.7\%]). } \\
N=288 \text { patients } \\
(n=186 \text { intervention } \\
\text { arm vs. } n=102 \text { control } \\
\text { arm) }\end{array}$ & $\begin{array}{c}\text { ESAS symptoms (pain, } \\
\text { tiredness, nausea, } \\
\text { depression, anxiety, } \\
\text { drowsiness, appetite } \\
\text { loss, well-being, and } \\
\text { shortness of breath). } \\
\text { Symptoms were } \\
\text { collected by a LHW } \\
\text { (weekly for high-risk } \\
\text { and monthly for } \\
\text { low-risk patients) by } \\
\text { telephone. A physician } \\
\text { assistant reviewed } \\
\text { symptoms daily and } \\
\text { notified the oncology } \\
\text { provider. }\end{array}$ & $\begin{array}{l}\text { Patient-reported } \\
\text { outcome: patients' } \\
\text { satisfaction with care } \\
\text { (question no. 18) } \\
\text { and self-reported } \\
\text { health status } \\
\text { (questions } \\
\text { no. } 23 \text { and } 24 \text { ) } \\
\text { of the Medicare } \\
\text { Advantage } \\
\text { and Prescription Drug } \\
\text { Consumer Assessment } \\
\text { of Healthcare } \\
\text { Providers and Systems. } \\
\text { Use of resources: } \\
\text { emergency department } \\
\text { visits, hospital } \\
\text { admissions, (including } \\
\text { readmissions and } \\
\text { intensive care unit } \\
\text { hospitalizations), and } \\
\text { the use of hospice } \\
\text { services. Median cost } \\
\text { per patient (12 months) } \\
\text { Clinical outcome: } \\
\text { survival during } \\
\text { follow-up (12 months) }\end{array}$ & 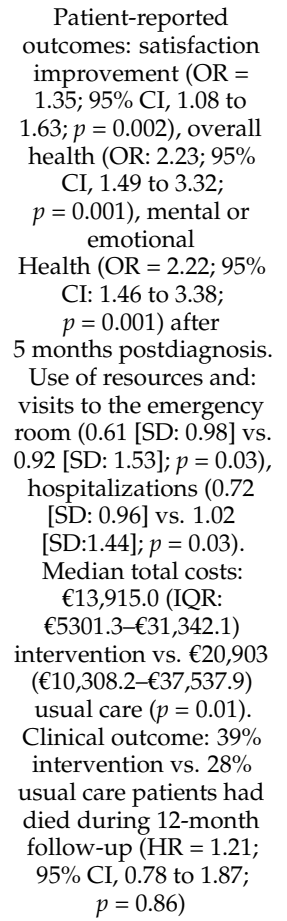 & $\begin{array}{l}\text { The LHW-led symptom } \\
\text { screening intervention } \\
\text { among patients with } \\
\text { advanced stages of } \\
\text { cancer was associated } \\
\text { with improved } \\
\text { patient-reported } \\
\text { outcomes, no } \\
\text { differences in survival, } \\
\text { and reduced acute } \\
\text { care use and total } \\
\text { health care costs. }\end{array}$ & 18/22 (STROBE) \\
\hline
\end{tabular}


Table 2. Cont.

\begin{tabular}{|c|c|c|c|c|c|c|c|c|}
\hline $\begin{array}{l}\text { Author and Year of } \\
\text { Publication, Country }\end{array}$ & $\begin{array}{l}\text { Type of Study (a), } \\
\text { Comparators (b) }\end{array}$ & Study Aim & $\begin{array}{l}\text { Cancer Type; } \\
\text { Sample Size }\end{array}$ & $\begin{array}{l}\text { Patient-Reported } \\
\text { Symptoms Assessed, } \\
\text { Instrument Used and } \\
\text { Frequency }\end{array}$ & $\begin{array}{l}\text { Type of Outcome } \\
\text { Evaluated }\end{array}$ & Summary of Results & Main Conclusion & Study Quality \\
\hline $\begin{array}{l}\text { De Raaf et al. [24], 2013, } \\
\text { The Netherlands }\end{array}$ & $\begin{array}{c}\text { (a) Randomized } \\
\text { non-blinded controlled } \\
\text { trial } \\
\text { (b) Protocolized } \\
\text { patient-tailored } \\
\text { treatment (PPT) of } \\
\text { physical symptoms vs. } \\
\text { care as usual (CAU) }\end{array}$ & $\begin{array}{l}\text { To investigate whether } \\
\text { monitoring of physical } \\
\text { symptoms coordinated } \\
\text { by a nurse has a more } \\
\text { favorable effect on } \\
\text { fatigue severity than } \\
\text { the symptom } \\
\text { management included } \\
\text { in the standard } \\
\text { oncologic care of } \\
\text { patients with advanced } \\
\text { cancer. }\end{array}$ & $\begin{array}{c}\text { Patients with solid } \\
\text { malignancies in } \\
\text { palliative care and } \\
\text { fatigued } \\
\text { Most prevalent: breast } \\
(36.8 \%), \text { gastrointestinal } \\
(30.9 \%) \text { and urogenital } \\
\text { cancer }(15.8 \%) \\
N=152 \text { patients }(n=76 \\
\text { in the PPT vs. } n=76 \text { in } \\
\text { CAU) }\end{array}$ & $\begin{array}{l}\text { Nine symptoms } \\
\text { (pain, nausea, vomiting, } \\
\text { constipation, diarrhea, } \\
\text { lack of appetite, } \\
\text { shortness of breath, } \\
\text { cough, and dry mouth) } \\
\text { Collected during } \\
\text { meetings with the } \\
\text { nurse specialist at the } \\
\text { outpatient clinic. When } \\
\text { patients rated a certain } \\
\text { symptom } \geq 4 \text { (from } 0 \\
\text { to } 10 \text { ), the nurses asked } \\
\text { the oncologist to start } \\
\text { an appropriate } \\
\text { treatment using specific } \\
\text { protocols. }\end{array}$ & $\begin{array}{c}\text { Patient-reported } \\
\text { outcome: fatigue (MFI } \\
\text { scores at T1 =1 month; } \\
\text { T2 = } 2 \text { months; T3 = } \\
\text { months after random } \\
\text { assignment). } \\
\text { Influence of fatigue on } \\
\text { daily life (BFI-I). } \\
\text { Anxiety and depressed } \\
\text { mood (HADS). }\end{array}$ & $\begin{array}{c}\text { Patient-reported } \\
\text { outcome: MFI scores: } \\
\text { T1 (mean } \\
\text { difference, }-0.84 \text { [SE: } \\
0.31] ; p=0.007), \mathrm{T} 2 \\
\text { (mean } \\
\text { difference, }-1.14 \text { [SE: } \\
0.40] ; p=0.005 \text { ), T3 } \\
\text { (mean difference, - } 0.90 \\
\text { [SE, } 0.50] ; p=0.07) \\
\text { Interference of } \\
\text { fatigue with daily life: } \\
\text { the PPT group reported } \\
\text { a decrease in the } \\
\text { interference of } \\
\text { fatigue with daily life } \\
\text { (maximal effect size, } \\
\text { 0.64; } p<0.001 \text { ) } \\
\text { Anxiety: anxiety } \\
\text { decreased in the PPT } \\
\text { group as compared } \\
\text { with the CAU group } \\
\text { (maximal effect size, } \\
0.32 ; p=0.001\end{array}$ & $\begin{array}{l}\text { In fatigued patients } \\
\text { with advanced cancer, } \\
\text { nurse-led monitoring } \\
\text { and protocolized } \\
\text { treatment of physical } \\
\text { symptoms are effective } \\
\text { in alleviating fatigue }\end{array}$ & 29/30 (CONSORT) \\
\hline $\begin{array}{c}\text { Diplock et al. [30], 2019, } \\
\text { Canada }\end{array}$ & $\begin{array}{c}\text { (a) Prospective } \\
\text { observational study } \\
\text { (b) Cancer patients } \\
\text { screened prior to and } \\
\text { after ESAS } \\
\text { implementation }\end{array}$ & $\begin{array}{l}\text { To assess the impact of } \\
\text { implementing ESAS } \\
\text { screening on HRQoL } \\
\text { and patient satisfaction } \\
\text { with care in ambulatory } \\
\text { oncology patients. }\end{array}$ & $\begin{array}{c}\text { Ambulatory oncology } \\
\text { patients. Most } \\
\text { prevalent: breast } \\
(25.4 \%) \text {, hematologic } \\
\text { oncology }(16.8 \%) \text { and } \\
\text { head and neck cancer } \\
(16.4 \%) \\
N=268 \text { patients } \\
(n=160 \text { prior to ESAS } \\
\text { site implementation vs. } \\
n=108 \text { after ESAS } \\
\text { implementation })\end{array}$ & $\begin{array}{c}\text { ESAS symptoms (pain, } \\
\text { tiredness, nausea, } \\
\text { depression, anxiety, } \\
\text { drowsiness, appetite } \\
\text { loss, well-being, and } \\
\text { shortness of breath). } \\
\text { ESAS was collected via } \\
\text { a touch screen kiosk (at } \\
\text { baseline } \\
\text { (T1), and two weeks } \\
\text { later (T2)) }\end{array}$ & $\begin{array}{c}\text { Patient-reported } \\
\text { outcome: HRQDLL } \\
\text { (EORTC-QLQ-C30). } \\
\text { Patients' satisfaction } \\
\text { with care } \\
\text { (PMH/PSQ-MD-24 and } \\
\text { EORTC-OUTPATSAT35 } \\
\text { RT and CT) }\end{array}$ & $\begin{array}{l}\text { No significant } \\
\text { differences between } \\
\text { HRQoL and } \\
\text { satisfaction outcomes } \\
\text { from the matched } \\
\text { non-ESAS and ESAS } \\
\text { groups (at T1 and T2) } \\
\text { Nausea and Vomiting } \\
\text { punctuations } \\
\text { significantly decreased } \\
\text { over time: mean } 10.75 \\
\text { (SD: 19.40) prior to } \\
\text { ESAS, mean 7.44 (SD: } \\
\text { 12.96) at T1 and mean } \\
\text { 8.13 (SD: } 14.64 \text { ) at T2 } \\
\text { Constipation: mean } \\
\text { 28.08 (SD: 32.23) prior } \\
\text { to ESAS, mean 13.28 } \\
\text { (SD: 21.51) at T1 and } \\
\text { mean 10.60 (SD: 18.50) } \\
\text { at T2 }\end{array}$ & $\begin{array}{l}\text { There was no impact of } \\
\text { early-ESAS screening } \\
\text { on HRQoL or } \\
\text { satisfaction outcomes }\end{array}$ & 16/22 (STROBE) \\
\hline
\end{tabular}


Table 2. Cont.

\begin{tabular}{|c|c|c|c|c|c|c|c|c|}
\hline $\begin{array}{l}\text { Author and Year of } \\
\text { Publication, Country }\end{array}$ & $\begin{array}{l}\text { Type of Study (a), } \\
\text { Comparators (b) }\end{array}$ & Study Aim & $\begin{array}{l}\text { Cancer Type; } \\
\text { Sample Size }\end{array}$ & $\begin{array}{c}\text { Patient-Reported } \\
\text { Symptoms Assessed, } \\
\text { Instrument Used and } \\
\text { Frequency }\end{array}$ & $\begin{array}{l}\text { Type of Outcome } \\
\text { Evaluated }\end{array}$ & Summary of Results & Main Conclusion & Study Quality \\
\hline $\begin{array}{l}\text { Baratelli et al. [31], } \\
\text { 2019, Italy }\end{array}$ & $\begin{array}{l}\text { (a) Observational, } \\
\text { prospective cohort } \\
\text { (b) Usual care vs. } \\
\text { patient-reported } \\
\text { symptoms }\end{array}$ & $\begin{array}{l}\text { To compare two groups } \\
\text { of patients: a first } \\
\text { group, visited using the } \\
\text { usual modality of } \\
\text { toxicity and } \\
\text { symptoms collection } \\
\text { and management, and } \\
\text { a second group, visited } \\
\text { after the introduction of } \\
\text { patient-based } \\
\text { assessment of } \\
\text { symptoms and } \\
\text { toxicities in routine } \\
\text { clinical practice }\end{array}$ & $\begin{array}{l}\text { Cancer patients } \\
\text { receiving active } \\
\text { anti-cancer treatment } \\
\text { as outpatients. } \\
\text { Most prevalent } \\
\text { colorectal cancer } \\
(32.7 \%), \text { lung cancer } \\
(19.9 \%) \text {, and pancreatic } \\
\text { cancer }(14.7 \%) \\
N=211(n=119 \text { usual } \\
\text { care vs. } n=92 \\
\text { patient-reported } \\
\text { symptoms patients) }\end{array}$ & $\begin{array}{c}\text { Thirteen symptoms } \\
\text { (mouth problems, } \\
\text { nausea, } \\
\text { vomiting, constipation, } \\
\text { diarrhea, dyspnea, skin } \\
\text { problems, nail } \\
\text { problems, itching, } \\
\text { hand/foot problems, } \\
\text { fatigue, pain, and other } \\
\text { issues) } \\
\text { Symptoms collected by } \\
\text { a nurse before each } \\
\text { visit and were paper } \\
\text { based. } \\
\text { They were delivered } \\
\text { to the physician, who } \\
\text { could consult them } \\
\text { before the visit. }\end{array}$ & $\begin{array}{l}\text { Patient-reported } \\
\text { outcome: HRQoL } \\
\text { (mean change from } \\
\text { baseline to 1 month of } \\
\text { (EORTC-QLQ-C30) } \\
\text { scores) }\end{array}$ & $\begin{array}{l}\text { Patient-reported } \\
\text { outcome: mean change } \\
\text { from baseline of global } \\
\text { QoL was - } 1.68 \text { (SE: } \\
\text { 1.88) for usual care vs. } \\
\text { 2.54 (SE: } 2.32 \text { ) in } \\
\text { patient-reported } \\
\text { symptoms }(p=0.004) \text {. }\end{array}$ & $\begin{array}{l}\text { Introduction of } \\
\text { patient-reported } \\
\text { symptom monitoring } \\
\text { in clinical practice } \\
\text { produced a significant } \\
\text { QoL improvement } \\
\text { compared to the } \\
\text { traditional modality of } \\
\text { visit. }\end{array}$ & 17/22 (STROBE) \\
\hline $\begin{array}{l}\text { Strasser et al. [25] 2016, } \\
\text { Switzerland }\end{array}$ & $\begin{array}{c}\text { (a) Multicenter cluster } \\
\text { randomized clinical } \\
\text { trial } \\
\text { (b) Patient-reported } \\
\text { symptoms and } \\
\text { monitoring vs. usual } \\
\text { care }\end{array}$ & $\begin{array}{l}\text { To test the effects of the } \\
\text { E-MOSAIC } \\
\text { intervention } \\
\text { (patient-reported } \\
\text { symptoms and } \\
\text { monitoring) in patients } \\
\text { with incurable cancer } \\
\text { getting a new line of } \\
\text { chemotherapy with } \\
\text { palliative intent. }\end{array}$ & $\begin{array}{c}\text { Patients received } \\
\text { anticancer treatment } \\
\text { with palliative intent } \\
\text { Most prevalent } \\
\text { non-small-cell lung } \\
\text { cancer. }(18.9 \%), \\
\text { colorectal cancer } \\
(14.4 \%) \text { and breast } \\
\text { cancer }(10.2 \%) \\
N=264(n=119 \text { usual } \\
\text { care vs. } n=145 \\
\text { patient-reported } \\
\text { symptoms patients) }\end{array}$ & $\begin{array}{l}\text { ESAS symptoms, and } \\
\text { three additional } \\
\text { outcomes (estimated } \\
\text { nutritional intake, body } \\
\text { weight change, and } \\
\text { Karnofsky } \\
\text { performance) } \\
\text { Electronic } \\
\text { patient-reported } \\
\text { symptoms collected } \\
\text { weekly. In the } \\
\text { intervention group, } \\
\text { longitudinal } \\
\text { monitoring sheet was } \\
\text { printed and was } \\
\text { immediately given to } \\
\text { the oncologists. }\end{array}$ & $\begin{array}{c}\text { Patient-reported } \\
\text { outcome: HRQoL } \\
\text { (mean change from } \\
\text { baseline to } 6 \text { weeks of } \\
\text { (G-QoL) scores for } \\
\text { EORTC-QLQ-C30; } \\
\text { difference between the } \\
\text { arms) }\end{array}$ & $\begin{array}{l}\text { Patient-reported } \\
\text { outcome: the difference } \\
\text { in HRQoL between } \\
\text { arms was } 6.84(-1.65, \\
15.33)(p=0.1) \text { in favor } \\
\text { of the intervention arm }\end{array}$ & $\begin{array}{l}\text { Monitoring of patient } \\
\text { symptoms, clinical } \\
\text { syndromes, and their } \\
\text { management clearly } \\
\text { reduced patients' } \\
\text { symptoms, and QoL } \\
\text { (although the difference } \\
\text { was not statistically } \\
\text { significant) }\end{array}$ & 30/30 (CONSORT) \\
\hline
\end{tabular}


Table 2. Cont.

\begin{tabular}{|c|c|c|c|c|c|c|c|c|}
\hline $\begin{array}{l}\text { Author and Year of } \\
\text { Publication, Country }\end{array}$ & $\begin{array}{l}\text { Type of Study (a), } \\
\text { Comparators (b) }\end{array}$ & Study Aim & $\begin{array}{l}\text { Cancer Type; } \\
\text { Sample Size }\end{array}$ & $\begin{array}{c}\text { Patient-Reported } \\
\text { Symptoms Assessed, } \\
\text { Instrument Used and } \\
\text { Frequency }\end{array}$ & $\begin{array}{l}\text { Type of Outcome } \\
\text { Evaluated }\end{array}$ & Summary of Results & Main Conclusion & Study Quality \\
\hline $\begin{array}{l}\text { Kneuertz et al. [32] } \\
\text { 2020, USA }\end{array}$ & $\begin{array}{l}\text { (a) Observational } \\
\text { prospective pilot study } \\
\text { (b) One arm }\end{array}$ & $\begin{array}{l}\text { To understand the } \\
\text { utility of a mobile } \\
\text { application platform to } \\
\text { engage patients whilst } \\
\text { gathering data on } \\
\text { patient compliance, } \\
\text { perioperative } \\
\text { experience and } \\
\text { satisfaction. }\end{array}$ & $\begin{array}{l}\text { Patients diagnosed } \\
\text { with lung cancer who } \\
\text { were scheduled for } \\
\text { robotic surgery } \\
N=50 \text { patients }\end{array}$ & $\begin{array}{l}\text { Post-discharge recovery } \\
\text { assessment of pain, } \\
\text { anxiety and mood. } \\
\text { Social role functioning } \\
\text { and return to work } \\
\text { Collected daily (pain, } \\
\text { anxiety and mood) and } \\
\text { at days } 14 \text { and } 30 \\
\text { post-discharge (social } \\
\text { role functioning and } \\
\text { return to work) } \\
\text { through a mobile } \\
\text { application. The care } \\
\text { team had access to } \\
\text { patients' reports. }\end{array}$ & $\begin{array}{l}\text { Patient-reported } \\
\text { outcome: satisfaction } \\
\text { with their hospital stay } \\
\text { (ad-hoc questionnaire) }\end{array}$ & $\begin{array}{l}\text { Patient-reported } \\
\text { outcome: } 77.4 \% \text { of } \\
\text { patients gave the } \\
\text { highest-ranking } \\
\text { ("excellent") for the } \\
\text { care received, and } \\
93.5 \% \text { reported they } \\
\text { would recommend the } \\
\text { hospital to others based } \\
\text { on their experience }\end{array}$ & $\begin{array}{c}\text { A mobile device } \\
\text { platform may serve as } \\
\text { an effective mechanism } \\
\text { to record perioperative } \\
\text { patient-reported } \\
\text { symptoms and } \\
\text { satisfaction while } \\
\text { facilitating } \\
\text { patient-provider } \\
\text { engagement in } \\
\text { perioperative care. }\end{array}$ & 17/22 (STROBE) \\
\hline $\begin{array}{l}\text { Riis et al. [26], 2020, } \\
\text { Denmark }\end{array}$ & $\begin{array}{l}\text { (a) Pilot randomized } \\
\text { controlled trial. } \\
\text { (b) standard follow-up } \\
\text { care vs. individualized } \\
\text { follow-up care }\end{array}$ & $\begin{array}{l}\text { To evaluate the patients' } \\
\text { satisfaction with the } \\
\text { care provided when } \\
\text { using electronic } \\
\text { patient-reported } \\
\text { symptom monitoring to } \\
\text { individualize follow-up } \\
\text { care in women with } \\
\text { early breast cancer } \\
\text { receiving adjuvant } \\
\text { endocrine therapy. }\end{array}$ & $\begin{array}{c}\text { Postmenopausal } \\
\text { women with early } \\
\text { breast cancer receiving } \\
\text { adjuvant endocrine } \\
\text { therapy. } \\
N=134(n=64 \\
\text { standard follow-up } \\
\text { care vs. } n=60 \\
\text { individualized } \\
\text { follow-up care) }\end{array}$ & $\begin{array}{l}\text { Quality of life (EORTC } \\
\text { QLQ-C30), including } \\
\text { three symptom scales } \\
\text { (fatigue, nausea and } \\
\text { vomiting, and pain), } \\
\text { and six single items } \\
\text { (appetite loss, diarrhea, } \\
\text { dyspnea, constipation, } \\
\text { insomnia, financial } \\
\text { impact) } \\
\text { Collected } \\
\text { every third month over } \\
\text { a two-year period } \\
\text { electronically. The } \\
\text { principal investigator } \\
\text { monitored incoming } \\
\text { questionnaires. }\end{array}$ & $\begin{array}{l}\text { Patient-reported } \\
\text { outcomes: satisfaction } \\
\text { with the care provided } \\
\text { as measured by four } \\
\text { items from the PEQ. } \\
\text { Secondary outcomes } \\
\text { were use of } \\
\text { consultations and } \\
\text { adherence to treatment } \\
\text { (collected every third } \\
\text { month over a two-year } \\
\text { period. }\end{array}$ & $\begin{array}{l}\text { Satisfaction with } \\
\text { follow-up care and } \\
\text { adherence: no } \\
\text { statistically significant } \\
\text { differences between } \\
\text { standard follow-up } \\
\text { care vs. individualized } \\
\text { follow-up care. } \\
\text { Use of resources: } \\
\text { patients in standard } \\
\text { care attended } 4.3 \text { (CI: } \\
\text { 3.9-4.7) consultations } \\
\text { each vs. } 2.1 \text { (CI: } 1.6-2.6) \\
\text { in patients attending } \\
\text { individualized care } \\
(p<0.001) .\end{array}$ & $\begin{array}{c}\text { A significant reduction } \\
\text { in consultations was } \\
\text { observed for the group } \\
\text { attending } \\
\text { individualized care } \\
\text { without compromising } \\
\text { the patients' } \\
\text { satisfaction, quality of } \\
\text { life, or adherence to } \\
\text { treatment }\end{array}$ & 24/30 (CONSORT) \\
\hline $\begin{array}{l}\text { Nipp et al. [27], 2019, } \\
\text { USA }\end{array}$ & $\begin{array}{l}\text { (a) Nonblinded, pilot } \\
\text { randomized controlled } \\
\text { trial. } \\
\text { (b) Usual care vs. } \\
\text { patient-reported } \\
\text { symptom monitoring. }\end{array}$ & $\begin{array}{l}\text { To assess the feasibility } \\
\text { and preliminary } \\
\text { efficacy of symptom } \\
\text { monitoring for } \\
\text { improving symptom } \\
\text { burden and health care } \\
\text { utilization among } \\
\text { hospitalized patients } \\
\text { with advanced cancer. }\end{array}$ & $\begin{array}{c}\text { Hospitalized patients } \\
\text { with advanced cancer } \\
\text { admitted to oncology } \\
\text { service. Most prevalent: } \\
\text { gastrointestinal }(36.6 \%), \\
\text { lung }(22 \%) \text { and head } \\
\text { and neck cancer }(10 \%) \\
N=150 \text { patients }(n=75 \\
\text { in the patient-reported } \\
\text { symptoms monitoring } \\
\text { vs. } n=75 \text { in usual care } \\
\text { arm) }\end{array}$ & $\begin{array}{l}\text { Physical symptoms } \\
\text { (pain, fatigue, } \\
\text { drowsiness, nausea, } \\
\text { appetite loss, dyspnea, } \\
\text { constipation, and } \\
\text { diarrhea) Psychological } \\
\text { symptoms (depression, } \\
\text { anxiety, and well-being) } \\
\text { Collected daily using } \\
\text { tablet computers. } \\
\text { Study staff presented } \\
\text { patients' reports each } \\
\text { day to the clinical staff. }\end{array}$ & $\begin{array}{l}\text { Use of resources and } \\
\text { cost outcomes: hospital } \\
\text { length of stay, time to } \\
\text { first unplanned } \\
\text { readmission within } \\
30 \text { days. }\end{array}$ & $\begin{array}{c}\text { No significant } \\
\text { difference in patients' } \\
\text { hospital length of stay } \\
(\mathrm{B}=0.16,95 \% \mathrm{CI} \\
-1.67-1.99 ; p=0.862) \text {. } \\
\text { Patients assigned to } \\
\text { symptom monitoring } \\
\text { had a lower risk of } \\
\text { readmissions } \\
(\mathrm{HR}=0.68,95 \% \mathrm{CI} \text { : } \\
0.37-1.26 ; p=0.224) .\end{array}$ & $\begin{array}{l}\text { Intervention patients } \\
\text { had lower readmission } \\
\text { risk, although this } \\
\text { difference was not } \\
\text { significant. }\end{array}$ & $27 / 30$ (CONSORT) \\
\hline
\end{tabular}


Table 2. Cont.

\begin{tabular}{|c|c|c|c|c|c|c|c|c|}
\hline $\begin{array}{l}\text { Author and Year of } \\
\text { Publication, Country }\end{array}$ & $\begin{array}{l}\text { Type of Study (a), } \\
\text { Comparators (b) }\end{array}$ & Study Aim & $\begin{array}{l}\text { Cancer Type; } \\
\text { Sample Size }\end{array}$ & $\begin{array}{c}\text { Patient-Reported } \\
\text { Symptoms Assessed, } \\
\text { Instrument Used and } \\
\text { Frequency }\end{array}$ & $\begin{array}{c}\text { Type of Outcome } \\
\text { Evaluated }\end{array}$ & Summary of Results & Main Conclusion & Study Quality \\
\hline $\begin{array}{l}\text { Howell et al. [33], 2020, } \\
\text { Canada }\end{array}$ & $\begin{array}{l}\text { (a) Observational } \\
\text { prospective study } \\
\text { (b) Usual care vs. } \\
\text { patient-reported } \\
\text { symptom monitoring }\end{array}$ & $\begin{array}{l}\text { To determine if } \\
\text { there was a difference } \\
\text { in relative rates for } \\
\text { emergency } \\
\text { department visits and } \\
\text { hospitalizations for the } \\
\text { iPEHOC (Improving } \\
\text { Patient Experience and } \\
\text { Health Outcomes } \\
\text { Collaborative } \\
\text { intervention) exposed } \\
\text { population compared } \\
\text { with contemporaneous } \\
\text { controls }\end{array}$ & $\begin{array}{c}\text { Different types of } \\
\text { cancer. Most prevalent: } \\
\text { prostate cancer }(16 \%), \\
\text { breast cancer }(14.9 \%), \\
\text { and gynecological } \\
\text { cancer }(8.7 \%) \\
N=129,797 \\
\text { (pre-intervention } \\
n=70,854 \text { and } \\
\text { intervention group } \\
n=58,943 \text { ) }\end{array}$ & $\begin{array}{l}\text { The iPEHOC } \\
\text { intervention included } \\
\text { patient-reported } \\
\text { symptoms collection } \\
\text { through an electronic } \\
\text { system in addition to } \\
\text { clinicians' and patients' } \\
\text { educational } \\
\text { interventions. } \\
\text { ESAS symptoms (pain, } \\
\text { tiredness, nausea, } \\
\text { depression, anxiety, } \\
\text { drowsiness, appetite } \\
\text { loss, well-being, and } \\
\text { shortness of breath) } \\
\text { In addition, Brief Pain } \\
\text { Inventory, the Cancer } \\
\text { Fatigue Scale, the } \\
\text { Generalized Anxiety } \\
\text { Disorder, and the } \\
\text { PHQ-9 }\end{array}$ & $\begin{array}{l}\text { Use of resources and } \\
\text { cost outcomes: } \\
\text { emergency department } \\
\text { visits, hospitalization } \\
\text { rates and drug } \\
\text { prescriptions for each } \\
\text { clinic (expressed as } \\
\text { difference in difference } \\
\text { (DID) approach). }\end{array}$ & $\begin{array}{c}\text { Use of resources: } \\
\text { DID =-0.223 in the RR } \\
\text { for emergency } \\
\text { department visits for } \\
\text { the intervention } \\
\text { compared with controls } \\
\text { over time }(0.947, \text { CI } \\
0.900-0.996) \text {. } \\
\text { There was also lower } \\
\text { DID in palliative care } \\
\text { visits }(-0.0097), \\
\text { psychosocial oncology } \\
\text { visits }(-0.0248 \text { and } \\
\text { antidepressant } \\
\text { prescriptions in the } \\
\text { exposed population } \\
\text { compared with } \\
\text { controls. }\end{array}$ & $\begin{array}{l}\text { Facilitating uptake of } \\
\text { patient-reported } \\
\text { symptoms data may } \\
\text { impact healthcare } \\
\text { utilization. }\end{array}$ & 19/22 (STROBE) \\
\hline
\end{tabular}


Table 2. Cont.

\begin{tabular}{|c|c|c|c|c|c|c|c|c|}
\hline $\begin{array}{l}\text { Author and Year of } \\
\text { Publication, Country }\end{array}$ & $\begin{array}{l}\text { Type of Study (a), } \\
\text { Comparators (b) }\end{array}$ & Study Aim & $\begin{array}{l}\text { Cancer Type; } \\
\text { Sample Size }\end{array}$ & $\begin{array}{c}\text { Patient-Reported } \\
\text { Symptoms Assessed, } \\
\text { Instrument Used and } \\
\text { Frequency }\end{array}$ & $\begin{array}{l}\text { Type of Outcome } \\
\text { Evaluated }\end{array}$ & Summary of Results & Main Conclusion & Study Quality \\
\hline $\begin{array}{l}\text { Nixon et al. [34] 2018, } \\
\text { Canada }\end{array}$ & $\begin{array}{c}\text { (a) Economic } \\
\text { evaluation based on the } \\
\text { data from a } \\
\text { randomized } \\
\text { nonblinded, clinical } \\
\text { trial } \\
\text { (b) Usual care vs. } \\
\text { patient-reported } \\
\text { symptom monitoring }\end{array}$ & $\begin{array}{l}\text { To evaluate the } \\
\text { cost-effectiveness of a } \\
\text { patient reported } \\
\text { outcome tool for } \\
\text { symptom monitoring } \\
\text { in patients undergoing } \\
\text { treatment for advanced } \\
\text { or metastatic } \\
\text { cancer compared to } \\
\text { standard of care } \\
\text { symptom monitoring } \\
\text { from the perspective of } \\
\text { the public payer in } \\
\text { Alberta. }\end{array}$ & $\begin{array}{l}\text { Patients with metastatic } \\
\text { solid tumors receiving } \\
\text { systemic therapy }\end{array}$ & $\begin{array}{l}\text { Twelve symptoms } \\
\text { (appetite loss, } \\
\text { constipation, cough, } \\
\text { diarrhea, dyspnea, } \\
\text { dysuria, fatigue, hot } \\
\text { flashes, nausea, pain, } \\
\text { neuropathy, and } \\
\text { vomiting) } \\
\text { Symptoms collected at } \\
\text { or between visits via a } \\
\text { web-based } \\
\text { questionnaire platform } \\
\text { (computer-experienced } \\
\text { patients) or } \\
\text { free-standing computer } \\
\text { kiosks (computer- } \\
\text { inexperienced patients). } \\
\text { The system included } \\
\text { email alerts to the } \\
\text { treating oncologist. }\end{array}$ & $\begin{array}{c}\text { Cost-utility analysis } \\
\text { (incremental } \\
\text { cost-effectiveness ratio } \\
\text { per life-year gained and } \\
\text { per QALY) }\end{array}$ & $\begin{array}{l}\text { The patient-reported } \\
\text { symptom monitoring } \\
\text { approach presented an } \\
\text { incremental } \\
\text { cost-effectiveness ratio } \\
\text { of } € 7575.9 \text { per QALY } \\
\text { (cost-effective) }\end{array}$ & $\begin{array}{l}\text { The use of a PRO tool } \\
\text { for symptom } \\
\text { monitoring yields a } \\
\text { cost per QALY of } \\
€ 7575.9 \text { that would be } \\
\text { considered a good } \\
\text { value for money at the } \\
\text { typically accepted } \\
\text { Canadian standard of } \\
\$ 50,000 \text { ( } € 28,163.2) \text { per } \\
\text { QALY }\end{array}$ & $\begin{array}{c}21 / 24 \\
\text { (CHEERS) }\end{array}$ \\
\hline
\end{tabular}

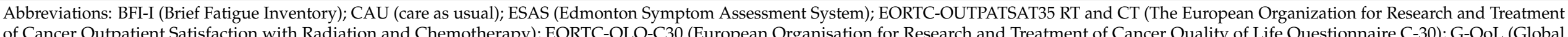

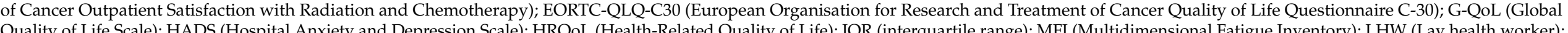

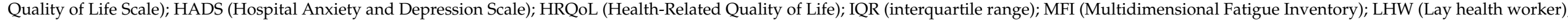

$\mathrm{PeQ}$ (patient experience questionnaire); PPT (patient-tailored treatment of physical symptoms); QALY (Quality-Adjusted Life Year). 


\subsection{Impact of Patient-Reported Symptoms on Health Outcomes}

Of the 16 studies identified, six [19-21,23,28,29] assessed the impact of the integration of patient-reported symptom monitoring on survival; nine [20,21,24-26,29-32] evaluated the impact of patient-reported symptom monitoring on other patient-reported outcomes such as HRQoL and satisfaction with treatment, and eight $[20-22,26,27,29,33,34]$ assessed economic outcomes, for example, the use of resources and costs of a patient-reported symptom monitoring strategy versus usual symptom monitoring.

\subsubsection{Overall Survival}

Six out of the 16 publications assessed the effects of patient-reported symptom monitoring on cancer patients' overall survival [19-21,23,28,29]. One of them [21] was a secondary analysis of data to explore the effect of age on different outcomes, including survival. All the studies, except for one registry [29], showed that patient-reported symptom surveillance led to significantly improved survival compared to usual care symptom monitoring. The clinical trial reported by Basch et al. [20], which included patients initiating chemotherapy for solid metastatic tumors $(N=766)$-mainly prostate $(17.4 \%)$, breast $(14.5 \%)$, and hematologic neoplasms (13.4\%)-reported an overall survival benefit of $6 \%$ (percentage of patients alive: $75 \%$ for patient-reported symptom monitoring vs. $69 \%$ for usual care; $p=0.05$ ) at one year of follow-up. Long-term results of the same trial [19] showed a gain of 5.2 months in favor of the patient-reported symptom monitoring arm in a median follow-up of seven years (median overall survival: 31.2 months (95\% CI, 24.5-39.6) vs. 26.0 months (95\% CI, 22.1-30.9) for usual care; $p=0.03$ ). Additionally, Nipp et al. [21] conducted a secondary analysis on data from Basch et al. $[19,20]$. They found that the survival benefits associated with patient-reported symptom surveillance were limited to younger patients ( $<70$ years): thus, the authors recorded a significant decrease in the hazard for death for patient-reported symptom monitoring ( $\mathrm{HR}=0.76 ; p=0.011)$ among younger patients ( $<70$ years); however, they did not find significant survival benefits for older patients ( $\geq 70$ years) assigned to the intervention $\operatorname{arm}(\mathrm{HR}=1.06 ; p=0.753)$.

Denis et al. [23] reported an increase of almost eight months' survival, after two years of follow-up, for the patient-reported monitoring arm (median overall survival: 22.5 months vs. 14.9 months usual care; HR: 0.59 (95\% CI, 0.37-0.96); $p=0.03)$ in a cohort of lung cancer patients $(N=121)$ with advanced nonprogressive disease (stages IIA to IV). In turn, the study of Barbera et al. [28] reported an absolute overall survival benefit at one year of $5.5 \%$ for patients exposed to patient-reported symptom monitoring vs. usual care (survival probability of $81.9 \%$ vs. $76.4 \% ; p<0.0001$ ) after one-year follow-up.

\subsubsection{Health-Related Quality of Life (HRQoL)}

Five studies evaluated the impact of patient-reported symptom surveillance on HRQoL $[20,21,25,30,31]$. The questionnaires used to assess HRQoL were the generic EuroQol 5-Dimensional Scale (EQ-5D) [20,21] or the cancer-specific Organization for Research and Treatment of The Cancer Quality of Life Questionnaire (EORTC-QLQ-C30) [25,30,31]. Most of the studies demonstrated how the interventions improved HRQoL compared to usual symptom monitoring. In the study by Basch et al. [20] in metastatic patients, the percentage of patients who registered a clinically meaningful HRQoL improvement ( $\geq 6$ points) after six months was significantly higher in the patient-reported symptoms arm than in the usual care arm $(21 \%$ vs. $11 \% ; p<0.001)$. A secondary analysis [21] showed that the HRQoL benefit obtained from electronic symptom monitoring did not change as a function of age $(\mathrm{B}=-0.02 ; p=0.994)$. Baratelli et al. [31] demonstrated that the introduction of patient-reported symptoms collection led to a significant benefit in HRQoL in patients actively receiving anti-cancer treatment (mean global QoL change from baseline to one month of follow-up: -1.68 (SE: 1.88) for usual care vs. 2.54 (SE: 2.32) for patient-reported symptoms $(p=0.004))$. 
Similarly, Strasser et al. [25] determined a between-arm difference in HRQoL of 6.84 $(-1.65,15.33)(p=0.1)$ in favor of the intervention arm for a cohort of cancer patients in palliative care $(N=264)$.

In turn, Diplock et al. [30] observed that HRQoL remained unchanged two weeks after the implementation of a patient-reported symptom monitoring strategy on ambulatory oncology patients $(N=268)$ mainly diagnosed with breast $(25.4 \%)$, hematologic $(16.8 \%)$, and head and neck cancer $(16.4 \%)$. However, nausea, vomiting and constipation scores significantly decreased after patient-reported symptom monitoring: for nausea and vomiting, the mean was 7.44 (SD: 12.96) at baseline, and 8.13 (SD: 14.64) two weeks after patient-reported symptom monitoring; for constipation, the mean was 13.28 (SD: 21.51) at baseline, and 10.60 (SD: 18.50) two weeks after patient-reported symptom monitoring.

\subsubsection{Patient-Reported Satisfaction}

Four studies rated patients' levels of satisfaction with care [26,29,30,32]. One of them used an ad-hoc questionnaire [32] for this purpose. In contrast, Diplock et al. [30] applied the Princess Margaret Hospital Patient Satisfaction Questionnaire (PMH/PSQ-MD-24), and Riis et al. [26] employed the Patient Experience Questionnaire (PEQ) to assess satisfaction in postmenopausal women with early breast cancer. Patel et al. [29] assessed patients satisfaction with care using the "satisfaction with provider" item of the validated Medicare Advantage and Prescription Drug Consumer Assessment of Healthcare Providers and Systems. As a result, two reports showed that although patients were satisfied with the care received, non-statistically significant differences were observed when comparing patientreported symptom surveillance with usual care [26,30]. In contrast, the study of Patel et al. [29] demonstrated that compared with usual care, the introduction of an LHW-led symptom screening intervention involved a significant improvement in satisfaction with care $(\mathrm{OR}=1.35 ; 95 \% \mathrm{CI}, 1.08$ to $1.63 ; p=0.002)$

\subsubsection{Patient-Reported Fatigue}

One study aimed at palliative cancer patients found out how monitoring and protocolizing the management of patient-reported symptoms alleviated fatigue in cancer patients in palliative care $(N=152)$ [24]. Specifically, Multidimensional Fatigue Inventory (MFI) scores were significantly lower in the patient-reported symptom monitoring arm than those reported in usual care after one and two months of follow-up (mean difference, -0.84 [SE: 0.31 ] $p=0.007$ after one month and mean difference, -1.14 [SE: 0.40]; $p=0.005$ after two months). Additionally, patients in the patient-reported symptom monitoring group had a significant decrease in the interference of fatigue with daily life (maximal effect size, 0.64; $p<0.001)$.

\subsubsection{Adherence}

Of the 16 studies, only one dealt with the effects of patient-reported symptom followup on medication adherence in early breast cancer patients receiving adjuvant endocrine therapy $(N=134)$ [26]. However, no statistically significant differences were observed when comparing usual symptom follow-up care.

\subsubsection{Economic Outcomes}

Eight publications assessed the use of resources and economic impact of a patientreported symptom monitoring [20-22,26,27,29,33,34], mainly in advanced or metastatic cancer $[20-22,27,29,34]$.

They showed that patient-reported symptom monitoring led to a substantial decrease in healthcare usage compared to usual care. Visits to the emergency room and hospitalizations and readmissions were lower in the intervention group than in the usual care monitoring group. In the study by Basch et al. [20], the percentage of metastatic cancer patients that visited the emergency room and were hospitalized was lower in the patient-centered surveillance arm than in the usual care arm (34\% vs. $41 \%, p=0.02 ; 45 \%$ 
vs. $49 \%, p=0.08$, respectively), although differences in hospitalized patients were not significant. A secondary analysis of this clinical trial according to patients' age [21] showed that the median time spent in the emergency room was significantly lower for the patientreported symptoms arm in younger patients compared to the control arm (21.72 months vs. 50.73 months; $p=0.016$ ).

Riis et al. [26] reported fewer consultations per patient by patients attending individualized care compared to standard care (2.1 [CI: 1.6-2.6] vs. 4.3 [CI 3.9-4.7]; $p<0.001)$. In another report [27] focusing on hospitalized patients with advanced cancer $(N=150)$, patients assigned to symptom monitoring had a lower risk of readmissions $(\mathrm{HR}=0.68$, 95\% CI: 0.37-1.26; $p=0.224$ ). Howell at al. [33], who assessed differences in healthcare utilization in a large cohort of cancer patients $(N=129797)$ who were mainly diagnosed with prostate cancer (16\%), breast cancer $(14.9 \%)$, and gynecological cancer $(8.7 \%)$, showed fewer emergency department visits (difference in difference (DID) $=-0.223$ ), palliative care visits (DID $=-0.0097)$, psychosocial oncology visits (DID $=-0.0248$ ) for the intervention group compared with control subjects over time. In turn, an LHW-led symptom screening intervention [29] was associated with an approximately $30 \%$ reduction in emergency visits (0.61 [SD: 0.98 ] vs. 0.92 [SD: 1.53 ]; $p=0.03)$ and hospitalizations (0.72 (SD: $0.96)$ vs. 1.02 (SD: 1.44$) ; p=0.03$ ) than the standard of care. In addition, median total costs (including all inpatient and outpatient services for 12 months) were also significantly lower in the intervention group than in the usual symptom monitoring cohort (€13,915.0 [IQR: 5301.3-31,342.1] vs. €20,903 [€10,308.2-€37,537.9]; $p=0.01)$. In contrast, an economic evaluation [22] showed that the average annual cost was higher for the patient-reported symptom monitoring arm (average annual cost of $€ 2828.1 /$ year/patient) compared to controls ( $€ 1129.2$ /year/patient). However, when including its survival benefits [23], patient-reported symptom monitoring was found to be a cost-effective strategy (incremental cost-effectiveness ratio of $€ 10,500.9$ per life-year gained and $€ 18107.9$ per Quality-Adjusted Life Year [QALY]) from the French national health insurance perspective. Another cost-effectiveness analysis based on data from Basch et al. [20] trial in metastatic patients, demonstrated that the electronic patient-reported symptom monitoring strategy was a cost-effective alternative (incremental cost-effectiveness ratio of $€ 10,500.9$ per QALY) from the perspective of the public payer in Canada.

\section{Discussion}

Our systematic literature review shows that patient-reported symptom monitoringmainly electronic-based-provides substantial benefits in terms of clinical (overall survival), other patient-reported (such as HRQoL and satisfaction, etc.), and economic (use of healthcare resources, costs, cost-effectiveness, etc.) outcomes.

On the one hand, we found that active patient-reported monitoring is associated with increased survival equal to or greater than five months compared with usual care in advanced cancer. One hypothesis for this remarkable benefit is that patient-reported surveillance allows clinicians to both respond earlier to worsening symptoms and tumor recurrence through the email alerts [19-21,23]; thus, clinicians can perform rapid interventions that prevent complications, unexpected hospitalizations, or chemotherapy withdrawal. Indeed, improvements in symptom management may also allow patients to tolerate and, consequently, to benefit from chemotherapy for a longer time than when in usual care-for example, in the study conducted by Basch et al. [20], the mean treatment time in the intervention group was 8.2 months vs. 6.3 months in the usual care group; $p=0.002$.

Although survival benefits were robust and consistent across most of the studies, we acknowledge that extrapolating these findings to the general population may be risky. Firstly, most studies that demonstrated this benefit were placed in a single country and department [19-21]. Added to this, most of them were randomized controlled clinical trials [19-21,23], where patients' eligibility criteria were very strict and the interventions very well-defined. Moreover, the results from a secondary analysis by Basch et al. [21] 
pointed out that survival benefits might be restricted to younger patients ( $<70$ years). The authors speculated that for elderly patients, many other factors apart from symptom monitoring might influence survival, such as mobility, cognitive function, and the availability of social support. Additionally, elderly patients are more likely to be computer illiterate, which could compromise the effectiveness of this type of intervention. Finally, another point to consider is that the effect of patient-reported symptom monitoring on survival seems to be stronger in the first year after diagnosis or recurrence than in the following years. Accordingly, Barbera et al. [28] observed an absolute overall survival benefit of $5.5 \%$ the first year, whereas it was $2.2 \%$ after 3 years and $0.5 \%$ after 5 years since diagnosis or recurrence. Thus, the authors hypothesized that early symptom identification and treatment imply a more noticeable benefit to patients when their general health state is worse, which generally coincides with the emergence and the recurrence of the disease.

On the other hand, patient-reported symptom monitoring significantly enhanced different patient-reported outcomes compared to usual care. For instance, some studies observed an HRQoL improvement associated with this monitoring strategy. The main reason for the HRQoL improvement might be the reduction in the severity of the symptoms. For example, better pain control might result in a better trend in global HRQoL [31]. Only one of the studies [30] showed that HRQoL did not differ between patient-reported and usual monitoring. The absence of differences may be due to low sensitivity for detecting HRQoL changes in a short time period (two weeks) and limited statistical power due to the small sample size. Despite this limitation, the authors still reported a significant improvement in some symptoms, reflecting greater attention to physical symptoms after patient-reported symptom implementation. Additionally, most of the patients surveyed were highly satisfied with the patient-reported symptoms approach $[26,29,30,32]$, although no significant differences were found when comparing this monitoring strategy with usual care in two of the studies [26,30]. Despite not being significant, these results can be considered a positive outcome: they indicate that more individualized patient-reported monitoring does not compromise patients' satisfaction with care.

Finally, most studies showed that patient-reported symptom monitoring led to economic benefits-namely, substantial savings on healthcare use of resources-compared to usual care symptom follow-up $[20,21,26,27,33]$. These savings may be attributable mainly to better symptom control, which prevents unplanned emergency room visits $[20,27,29,33]$ and hospital readmissions [27,29].

With respect to medical costs, one observational study [29] demonstrated how an LHW-led symptom screening intervention significantly decreased healthcare costs, mainly due to reduced acute care. In contrast, Lizee et al. [22] showed that patient-reported symptom monitoring increased the average annual cost of surveillance, mainly due to the extra expenses associated with the electronic system. However, when adding the survival benefits associated with patient-reported symptom monitoring [23], the intervention proved to be cost-effective from the French national health insurance perspective. Despite this positive result, some study limitations must be taken into consideration: (1) the analysis was limited to the time horizon of the trial (two years follow-up); (2) the EQ-5D utilities were not measured in the trial and were derived from prior research; (3) the study was conducted from a payer's perspective (the authors did not consider societal costs [e.g., productivity costs or informal care]).

\subsection{Other Implications of Patient-Reported Symptom Monitoring}

Besides the benefits of survival, HRQoL, and economic outcomes already reported, monitoring patient-reported symptoms might also have other implications in clinical practice that deserve particular attention despite being beyond the scope of the present review. For instance, this systematic monitoring might make cancer patients more aware of their symptoms and disease as a whole [35-37]. Thus, patients can better inform their providers about their health status [37] and feel more comfortable initiating discussions with the medical team about their symptoms or other concerns $[38,39]$. Therefore, it 
seems that the systematic collection of patient-reported symptoms helps to build a better rapport and break down barriers in communication between patients and clinicians [40]. Better patient-physician communication eventually helps physicians to identify health problems that might otherwise go unnoticed [22,39], better focus the consultation, and devote more time to patients' main concerns [41]. Another interesting implication involves the increasing importance of telemedicine and electronic devices and software in current clinical practice, especially after the outbreak of the crisis caused by severe acute respiratory syndrome coronavirus 2 (SARS-CoV-2). In this regard, remote monitoring of patientreported symptoms provides an excellent opportunity to improve cancer management, reduce contact between individuals, and prevent unnecessary visits to hospital.

Despite the well-documented benefits associated with patient-reported symptom monitoring, significant limitations might still prevent its widespread implementation in clinical practice. The major barrier might be logistical, as many patients are unfamiliar with electronic devices or are computer illiterate, especially elderly patients. Thus, these groups of patients are at higher risk of being excluded from this technology. The second barrier is related to patients' compliance as they must be engaged to fill in the questionnaires regularly (sometimes every week). As a result, compliance might decrease over time. Lastly, monitoring of patient-reported symptoms may be a burden for care providers as it may increase care costs. For this reason, more cost-effectiveness studies should be conducted in different settings to demonstrate how the clinical benefits outweigh the possible extra costs associated with patient-reported active surveillance.

\subsection{Study Limitations}

Finally, some limitations of the present study should be considered. The first is related to its design. A systematic review examines and synthesizes the information on a subject available in the literature and includes some bias associated with the publications. The main biases associated with publications have already been considered throughout the discussion section. The second limitation concerns the research source and languages of publication as we have limited the search to one database and to publications in English and Spanish. We acknowledge that these restrictions may result in the omission of patientreported symptom monitoring interventions discussed in other databases or languages.

Although the benefits derived from patient-reported symptom surveillance are welldocumented and consistent across the different studies, a formal meta-analysis might be necessary to validate the effect of the symptom monitoring intervention on the different outcomes evaluated.

\section{Conclusions}

In this review, we have appraised recent evidence on the benefits of patient-reported symptom monitoring in cancer patients. The evidence illustrates that this monitoring modality entails an outstanding survival benefit for advanced cancer patients-five or more additional survival months than for usual care monitoring. Additionally, patient-reported symptom monitoring showed a positive effect on patients' HRQoL and satisfaction with care. It led to a substantial decrease in healthcare usage, preventing unplanned emergency room visits and hospital readmissions. Monitoring patient-reported symptoms might also have other implications in clinical practice that could be the subject of another study, such as promoting patients' disease awareness or improving patient-physician communication and relations.

Notwithstanding these advantages, there are still logistical barriers that prevent its widespread implementation - especially in its electronic modality. In addition, the realworld effectiveness and the cost-effectiveness of this strategy are yet to be proven in different settings. 
Supplementary Materials: The following are available online at https: / www.mdpi.com/article/ 10.3390/cancers13184615/s1, Table S1:PRISMA checklist, Table S2: PRISMA checklist for abstracts, Table S3: Comprehensive list of search terms considered, Table S4: Search strategy, Table S5: Excluded studies.

Author Contributions: Conceptualization, L.L., L.P.-C. and M.C.; methodology, L.L., L.P.-C. and M.C.; formal analysis, L.P.-C. and M.C.; data curation, L.P.-C. and M.C.; writing-original draft preparation, L.P.-C.; writing-review and editing, L.L. and M.C. All authors have read and agreed to the published version of the manuscript.

Funding: This research received no external funding.

Conflicts of Interest: The authors declare no conflict of interest.

\section{References}

1. Henry, D.H.; Viswanathan, H.N.; Elkin, E.P.; Traina, S.; Wade, S.; Cella, D. Symptoms and treatment burden associated with cancer treatment: Results from a cross-sectional national survey in the U.S. Support. Care Cancer 2008, 16, 791-801. [CrossRef]

2. Fromme, E.K.; Eilers, K.M.; Mori, M.; Hsieh, Y.C.; Beer, T.M. How accurate is clinician reporting of chemotherapy adverse effects? A comparison with patient-reported symptoms from the Quality-of-Life Questionnaire C30. J. Clin. Oncol. 2004, 22, 3485-3490. [CrossRef]

3. Laugsand, E.A.; Sprangers, M.A.; Bjordal, K.; Skorpen, F.; Kaasa, S.; Klepstad, P. Health care providers underestimate symptom intensities of cancer patients: A multicenter European study. Health Qual. Life Outcomes 2010, 8, 104. [CrossRef]

4. James, B.C.; Aschebrook-Kilfoy, B.; White, M.G.; Applewhite, M.K.; Kaplan, S.P.; Angelos, P.; Kaplan, E.L.; Grogan, R.H. Quality of life in thyroid cancer-assessment of physician perceptions. J. Surg. Res. 2018, 226, 94-99. [CrossRef]

5. Carlson, L.E.; Angen, M.; Cullum, J.; Goodey, E.; Koopmans, J.; Lamont, L.; MacRae, J.H.; Martin, M.; Pelletier, G.; Robinson, J.; et al. High levels of untreated distress and fatigue in cancer patients. Br. J. Cancer. 2004, 90, 2297-2304. [CrossRef] [PubMed]

6. Cleeland, C.S. Cancer-related symptoms. Semin. Radiat. Oncol. 2000, 10, 175-190. [CrossRef]

7. Eid, R.; Haddad, F.G.; Kourie, H.R.; Kattan, J. Electronic patient-reported outcomes: A revolutionary strategy in cancer care. Future Oncol. 2017, 13, 2397-2399. [CrossRef] [PubMed]

8. Riis, C.L.; Bechmann, T.; Jensen, P.T.; Coulter, A.; Steffensen, K.D. Are patient-reported outcomes useful in post-treatment follow-up care for women with early breast cancer? A scoping review. Patient Relat. Outcome Meas. 2019, 10, 117-127. [CrossRef]

9. Kotronoulas, G.; Kearney, N.; Maguire, R.; Harrow, A.; Di Domenico, D.; Croy, S.; MacGillivray, S. What is the value of the routine use of patient-reported outcome measures toward improvement of patient outcomes, processes of care, and health service outcomes in cancer care? A systematic review of controlled trials. J. Clin. Oncol. 2014, 32, 1480-1501. [CrossRef] [PubMed]

10. Howell, D.; Molloy, S.; Wilkinson, K.; Green, E.; Orchard, K.; Wang, K.; Liberty, J. Patient-reported outcomes in routine cancer clinical practice: A scoping review of use, impact on health outcomes, and implementation factors. Ann. Oncol. 2015, 26, 1846-1858. [CrossRef]

11. Chen, J.; Ou, L.; Hollis, S.J. A systematic review of the impact of routine collection of patient reported outcome measures on patients, providers and health organisations in an oncologic setting. BMC Health Serv. Res. 2013, 13, 211. [CrossRef]

12. Graupner, C.; Kimman, M.L.; Mul, S.; Slok, A.H.; Claessens, D.; Kleijnen, J.; Dirksen, C.D.; Breukink, S.O. Patient outcomes, patient experiences and process indicators associated with the routine use of patient-reported outcome measures (PROMs) in cancer care: A systematic review. Support. Care Cancer 2021, 29, 573-593. [CrossRef] [PubMed]

13. The Cochrane Collaboration. Cochrane Handbook for Systematic Reviews of Interventions. Available online: http:training. cochrane.org/handbook/archive/v5.1/ (accessed on 21 December 2020).

14. Page, M.J.; McKenzie, J.E.; Bossuyt, P.M.; Boutron, I.; Hoffmann, T.C.; Mulrow, C.D.; Shamseer, L.; Tetzlaff, J.M.; Akl, E.A.; Brennan, S.E.; et al. The PRISMA 2020 statement: An updated guideline for reporting systematic reviews. BMJ 2021, 372, n71. [CrossRef] [PubMed]

15. Schulz, K.F.; Altman, D.G.; Moher, D. CONSORT 2010 statement: Updated guidelines for reporting parallel group randomised trials. Int. J. Surg. 2011, 9, 672-677. [CrossRef] [PubMed]

16. Vandenbroucke, J.P.; Von Elm, E.; Altman, D.G.; Gøtzsche, P.C.; Mulrow, C.D.; Pocock, S.J.; Poole, C.; Schlesselman, J.J.; Egger, M.; Strobe Initiative. Strengthening the Reporting of Observational Studies in Epidemiology (STROBE): Explanation and elaboration. Ann. Intern. Med. 2007, 147, W163-W194. [CrossRef]

17. Husereau, D.; Drummond, M.; Petrou, S.; Carswell, C.; Moher, D.; Greenberg, D.; Augustovski, F.; Briggs, A.H.; Mauskopf, J.; Loder, E. Consolidated Health Economic Evaluation Reporting Standards (CHEERS) statement. Eur. J. Health Econ. 2013, 14, 367-372. [CrossRef]

18. The Campbell and Cochrane Economics Methods Group (CCEMG), Evidence for Polilcy and Practice Information and Coordinating Centre (EPPI-Centre). CCEMG -EPPI -Centre Cost Converter. Available online: http:/ / eppi.ioe.ac.uk/costconversion/ default.aspx (accessed on 27 May 2021).

19. Basch, E.; Deal, A.M.; Dueck, A.C.; Scher, H.I.; Kris, M.G.; Hudis, C.; Schrag, D. Overall Survival Results of a Trial Assessing Patient-Reported Outcomes for Symptom Monitoring During Routine Cancer Treatment. JAMA 2017, 318, 197-198. [CrossRef] 
20. Basch, E.; Deal, A.M.; Kris, M.G.; Scher, H.I.; Hudis, C.A.; Sabbatini, P.; Rogak, L.; Bennett, A.V.; Dueck, A.C.; Atkinson, T.M.; et al. Symptom Monitoring With Patient-Reported Outcomes During Routine Cancer Treatment: A Randomized Controlled Trial. J. Clin. Oncol. 2016, 34, 557-565. [CrossRef]

21. Nipp, R.D.; Horick, N.K.; Deal, A.M.; Rogak, L.J.; Fuh, C.; Greer, J.A.; Dueck, A.C.; Basch, E.; Temel, J.S.; El-Jawahri, A. Differential effects of an electronic symptom monitoring intervention based on the age of patients with advanced cancer. Ann. Oncol. 2020, 31, 123-130. [CrossRef]

22. Lizée, T.; Basch, E.; Trémolières, P.; Voog, E.; Domont, J.; Peyraga, G.; Urban, T.; Bennouna, J.; Septans, A.L.; Balavoine, M.; et al. Cost-Effectiveness of Web-Based Patient-Reported Outcome Surveillance in Patients With Lung Cancer. J. Thorac. Oncol. 2019, 14, 1012-1020. [CrossRef]

23. Denis, F.; Basch, E.; Septans, A.L.; Bennouna, J.; Urban, T.; Dueck, A.C.; Letellier, C. Two-Year Survival Comparing Web-Based Symptom Monitoring vs. Routine Surveillance Following Treatment for Lung Cancer. JAMA 2019, 321, 306-307. [CrossRef]

24. De Raaf, P.J.; de Klerk, C.; Timman, R.; Busschbach, J.J.; Oldenmenger, W.H.; van der Rijt, C.C. Systematic monitoring and treatment of physical symptoms to alleviate fatigue in patients with advanced cancer: A randomized controlled trial. J. Clin. Oncol. 2013, 31, 716-723. [CrossRef]

25. Strasser, F.; Blum, D.; Von Moos, R.; Cathomas, R.; Ribi, K.; Aebi, S.; Betticher, D.; Hayoz, S.; Klingbiel, D.; Brauchli, P.; et al. The effect of real-time electronic monitoring of patient-reported symptoms and clinical syndromes in outpatient workflow of medical oncologists: E-MOSAIC, a multicenter cluster-randomized phase III study (SAKK 95/06). Ann. Oncol. 2016, 27, 324-332. [CrossRef]

26. Riis, C.L.; Jensen, P.T.; Bechmann, T.; Möller, S.; Coulter, A.; Steffensen, K.D. Satisfaction with care and adherence to treatment when using patient reported outcomes to individualize follow-up care for women with early breast cancer-A pilot randomized controlled trial. Acta Oncol. 2020, 59, 444-452. [CrossRef] [PubMed]

27. Nipp, R.D.; El-Jawahri, A.; Ruddy, M.; Fuh, C.; Temel, B.; D’Arpino, S.M.; Cashavelly, B.J.; Jackson, V.A.; Ryan, D.P.; Hochberg, E.P.; et al. Pilot randomized trial of an electronic symptom monitoring intervention for hospitalized patients with cancer. Ann Oncol. 2019, 30, 274-280. [CrossRef]

28. Barbera, L.; Sutradhar, R.; Seow, H.; Mittmann, N.; Howell, D.; Earle, C.C.; Li, Q.; Thiruchelvam, D. The impact of routine Edmonton Symptom Assessment System (ESAS) use on overall survival in cancer patients: Results of a population-based retrospective matched cohort analysis. Cancer Med. 2020, 9, 7107-7115. [CrossRef] [PubMed]

29. Patel, M.I.; Ramirez, D.; Agajanian, R.; Agajanian, H.; Bhattacharya, J.; Bundorf, K.M. Lay Health Worker-Led Cancer Symptom Screening Intervention and the Effect on Patient-Reported Satisfaction, Health Status, Health Care Use, and Total Costs: Results From a Tri-Part Collaboration. JCO Oncol. Pract. 2020, 16, e19-e28. [CrossRef] [PubMed]

30. Diplock, B.D.; McGarragle, K.M.; Mueller, W.A.; Haddad, S.; Ehrlich, R.; Yoon, D.H.A.; Cao, X.; Al-Allaq, Y.; Karanicolas, P.; Fitch, M.I.; et al. The impact of automated screening with Edmonton Symptom Assessment System (ESAS) on health-related quality of life, supportive care needs, and patient satisfaction with care in 268 ambulatory cancer patients. Support. Care Cancer 2019, 27, 209-218. [CrossRef] [PubMed]

31. Baratelli, C.; Turco, C.G.C.; Lacidogna, G.; Sperti, E.; Vignani, F.; Marino, D.; Zichi, C.; De Luca, E.; Audisio, M.; Ballaminut, D.; et al. The role of patient-reported outcomes in outpatients receiving active anti-cancer treatment: Impact on patients' quality of life. Support. Care Cancer 2019, 27, 4697-4704. [CrossRef] [PubMed]

32. Kneuertz, P.J.; Jagadesh, N.; Perkins, A.; Fitzgerald, M.; Moffatt-Bruce, S.D.; Merritt, R.E.; D'Souza, D.M. Improving patient engagement, adherence, and satisfaction in lung cancer surgery with implementation of a mobile device platform for patient reported outcomes. J. Thorac. Dis. 2020, 12, 6883-6891. [CrossRef] [PubMed]

33. Howell, D.; Li, M.; Sutradhar, R.; Gu, S.; Iqbal, J.; O’Brien, M.A.; Seow, H.; Dudgeon, D.; Atzema, C.; Earle, C.C.; et al. Integration of patient-reported outcomes (PROs) for personalized symptom management in "real-world" oncology practices: A population-based cohort comparison study of impact on healthcare utilization. Support. Care Cancer 2020, 28, 4933-4942. [CrossRef]

34. Nixon NAS, E.; Clement, F.; Verma, S.; Manns, B. Cost-effectiveness of symptom monitoring with patient-reported outcomes during routine cancer treatment. J. Cancer Policy 2018, 15, 32-36. [CrossRef]

35. Kuijpers, W.; Groen, W.G.; Loos, R.; Oldenburg, H.S.; Wouters, M.W.; Aaronson, N.K.; van Harten, W.H. An interactive portal to empower cancer survivors: A qualitative study on user expectations. Support. Care Cancer 2015, 23, 2535-2542. [CrossRef]

36. Mooney, K.H.; Beck, S.L.; Friedman, R.H.; Farzanfar, R. Telephone-linked care for cancer symptom monitoring: A pilot study. Cancer Pract. 2002, 10, 147-154. [CrossRef]

37. Patel, R.A.; Klasnja, P.; Hartzler, A.; Unruh, K.T.; Pratt, W. Probing the benefits of real-time tracking during cancer care. AMIA Annu. Symp. Proc. 2012, 2012, 1340-1349. [PubMed]

38. Mark, T.L.; Fortner, B.; Johnson, G. Evaluation of a tablet PC technology to screen and educate oncology patients. Support. Care Cancer 2008, 16, 371-378. [CrossRef] [PubMed]

39. Takeuchi, E.E.; Keding, A.; Awad, N.; Hofmann, U.; Campbell, L.J.; Selby, P.J.; Brown, J.M.; Velikova, G. Impact of patient-reported outcomes in oncology: A longitudinal analysis of patient-physician communication. J. Clin. Oncol. 2011, 29, 2910-2917. [CrossRef] 
40. Velikova, G.; Keding, A.; Harley, C.; Cocks, K.; Booth, L.; Smith, A.B.; Wright, P.; Selby, P.J.; Brown, J.M. Patients report improvements in continuity of care when quality of life assessments are used routinely in oncology practice: Secondary outcomes of a randomised controlled trial. Eur. J. Cancer 2010, 46, 2381-2388. [CrossRef]

41. Lynch, J.; Goodhart, F.; Saunders, Y.; O'Connor, S.J. Screening for psychological distress in patients with lung cancer: Results of a clinical audit evaluating the use of the patient Distress Thermometer. Support. Care Cancer 2010, 19, 193-202. [CrossRef] [PubMed] 